\title{
Memoria histórica del Colegio Público de Esquipulas de Managua. Un proyecto de emprendimiento comunitario
}

\author{
Historical memory of the public school of Esquipulas de Managua. \\ A community entrepreneurship project
}

Julio César Orozco Alvarado ${ }^{1}$

Adolfo Alejandro Díaz Pérez ${ }^{2}$

\section{Resumen}

La memoria histórica del Colegio Público de Esquipulas es construida mediante la metodología de la historia oral, a partir de los testimonios de docentes, exalumnos, profesores jubilados, directores, administrativos, pobladores de la comunidad de Esquipulas, y de la revisión de documentos oficiales, libros de registros y de actas e informes que archiva el colegio. Los resultados que se presentan son producto de los aspectos esenciales de la historia del colegio, entre ellos: su origen, nombres qua ha tenido en su proceso histórico, el sueño del laboratorio de computación, las memorias y vivencias de los maestros, directores y personal administrativo. El artículo concluye haciendo una reflexión acerca de la importancia de la recuperación de la memoria histórica de los centros educativos como una tarea que también concierne al profesorado para el fortalecimiento de la identidad local e institucional y la promoción de valores como la pertenencia local y nacional, valores útiles en la preservación y cuido de la propiedad pública y privada.

Palabras clave: Colegio Público, Entrevista, Historia Oral, Memoria Histórica.

\section{Abstract}

The historical memory of the Esquipulas Public School is built using the methodology of oral history, based on the testimonies of teachers, alumni, retired teachers, directors, administrators, residents of the community of Esquipulas, and the review of official documents. books of records and minutes and reports that the school files. The results presented are the product of the essential aspects of the history of the school, including: its origin, names qua has had in its historical process, the dream of the computer lab, the memories and experiences of teachers, directors and

1 Doctor en Educación e Intervención Social. Profesor de la Facultad de Educación e Idiomas de la Universidad Nacional Autónoma de Nicaragua-Managua. Email: jorozcoa@hotmail.com ㄴ: https://orcid.org/oooo-0003-4819-0598

2 Licenciado en Ciencias de la Educación con mención en Ciencias Sociales. Profesor de la Facultad de Educación e Idiomas de la Universidad Nacional Autónoma de Nicaragua-Managua. Email: adolfoalejandro73@yahoo.com (D): https://orcid.org/o0oo-0002-4295-4094

Recibido: 03/04/2019 Aprobado: 22/05/2019 
staff administrative. The article concludes by reflecting on the importance of recovering the historical memory of schools as a task that also concerns teachers for the strengthening of local and institutional identity and the promotion of values such as local and national belonging, Useful values in the preservation and care of public and private property.

Key Words: Interview, Historical Memory, Public School, Oral History.

\section{Introducción}

El rescate de la memoria histórica sobre acontecimientos, experiencias o cualquier ámbito específico, es una de las tareas principales que se logra mediante la metodología de la historia oral. En el contexto educativo nicaragüense, es importante reconocer los valiosos aportes que a través de esta metodología se ha brindado para la reconstrucción de las historias de barrios, comunidades, personajes, prácticas culturales y acontecimientos sociopolíticos, sin embargo, es evidente que el uso de la historia oral en los procesos educativos es poco aplicado en la comunidad educativa.

A partir de esto, en el área de ciencias sociales de la Facultad de Educación e Idiomas de la Universidad Nacional Autónoma de Nicaragua (UNAN-Managua), inicia la línea de investigación de historia oral, cuyo propósito es brindar aportes sobre aspectos relacionados con la educación y las ciencias sociales, lo cual ha llevado a incursionar recientemente en la reconstrucción de las memorias históricas sobre personajes de la educación, historias de vida de profesores, memorias sobre los procesos de aprendizajes en las distintas épocas y, en este caso, la reconstrucción de la historia del Colegio Público de Esquipulas, del Distrito V de Managua.

Los resultados expuestos mediante el presente artículo -por razones de extensiónretoma los hallazgos más esenciales obtenidos durante el proceso de investigación, por lo que, únicamente se hará alusión a la fundación del colegio, la participación de la comunidad en la fundación del mismo, los nombres que ha tenido este centro educativo en toda su historia, la construcción del laboratorio de computación y las personalidades más recordadas de este centro educativo.

\section{Literatura}

En la historia del Colegio Público de Esquipulas están asociados una serie de conceptos que permiten comprender de manera más clara el contexto en que se dio el surgimiento de esta institución educativa. Para ello, a continuación se presentará un marco conceptual de los mismos:

Cáritas internacionales: La palabra Cáritas es un término que procede del latín Charitas (Caridad) y que significa amor. Así Cáritas es el organismo oficial de la Iglesia 
católica para la realización de la acción caritativa y social en sus diversos niveles: parroquial, diocesano regional y nacional, como expresión de la opción preferencial de la Iglesia por los más desfavorecidos.

Cáritas se dedica al combate contra la pobreza, la exclusión, la intolerancia y la discriminación, habilita a personas con menos recursos a participar en los asuntos que afectan directamente sus vidas e intercede por ellos en foros tanto nacionales como internacionales. Además, brinda ayuda a personas en riesgo de exclusión social, dándoles hogar por algunos días, alimento y servicios básicos de salud (PELLICER, 2000). En el caso de Nicaragua han financiado muchas obras sociales, el colegio público Esquipulas fue beneficiado para emprender la construcción de la primera fase del colegio, por lo cual tendrán hoy y siempre el agradecimiento de la comunidad de Esquipulas.

Instituto Nicaragüense de Acueductos y Alcantarillados (INAA): El instituto nicaragüense de acueductos y alcantarillados es un ente autónomo del Estado que depende jerárquicamente de la Presidencia de la República y fue creado mediante Decreto No. 20, publicado en La Gaceta, diario oficial No. 3 del 24 de agosto de 1979; atribuyéndole las funciones de: planear, diseñar, construir, operar, mantener, comercializar y administrar los acueductos y alcantarillados del país, para satisfacer las necesidades de agua potable y saneamiento de la población. Este instituto se hace mención en la narrativa de las entrevistas sobre la historia del colegio Esquipulas, este instituto era quien administraba la distribución del agua potable, y tuvo una propiedad con un tanque de agua para abastecer de agua potable a la comunidad de Esquipulas frente al colegio Esquipulas. En la narrativa esta propiedad pasa a manos del colegio Esquipulas por abandono de INNA.

Ministerio de Desarrollo Agropecuario y Reforma Agraria (MIDINRA): El CIERAMIDINRA, era un ministerio del gobierno de reconstrucción nacional, el cual fue fundado en 1979 al iniciar las funciones estatales el gobierno revolucionario, sus funciones era archivar las memorias de la administración de la reforma agraria, fomentar cursos de derecho agrario, realizar informe del estudio de la Reforma Agraria y Cooperativas en las regiones de Nicaragua, así como realizar estudios científicos de la agro tecnología y las estadísticas básicas en el sector agropecuario. Esta institución se relaciona con la Historia del Colegio porque poseía una propiedad de cinco manzanas en la comunidad de Esquipulas, la cual fue asignada al Colegio para la construcción de un Anexo, por medio de los Comités de Defensa Sandinistas (CDS).

\section{Metodología}

La investigación asume el paradigma interpretativo -también denominado "emergente, etnográfico, fenomenológico, hermenéutico, humanístico, interpretativo y naturalista" (Fernández y Rivera, 2015, p.93)- como un posicionamiento frente al 
objeto de investigación, el cual permite tratar los fenómenos sociales e interpretar y comprender lo que ocurre en diferentes contextos humanos. Desde esta perspectiva, el investigador tiene libertad para "describir los sucesos que ocurren en la vida de un grupo" (Martínez González, 2007, p.31) y "comprender, develar y describir una situación determinada" (Abraham, 2015, p.120).

Con base en estos referentes, la investigación se diseñó desde un enfoque cualitativo para "obtener las perspectivas y puntos de vistas de los participantes [recuerdos, emociones y experiencias]" y recopilar "las vivencias de los participantes tal como fueron (o son) sentidas y experimentadas" (Hernández, Fernández y Baptista, 2014, p.8). Asimismo, la investigación se adhirió al método de la historia oral, ya que éste es propicio para la "recopilación y recuperación de la memoria de aquellas personas que vivieron en otra época distinta a la nuestra, en un momento histórico concreto" (Rodríguez, Luque y Navas, 2014 p.194).

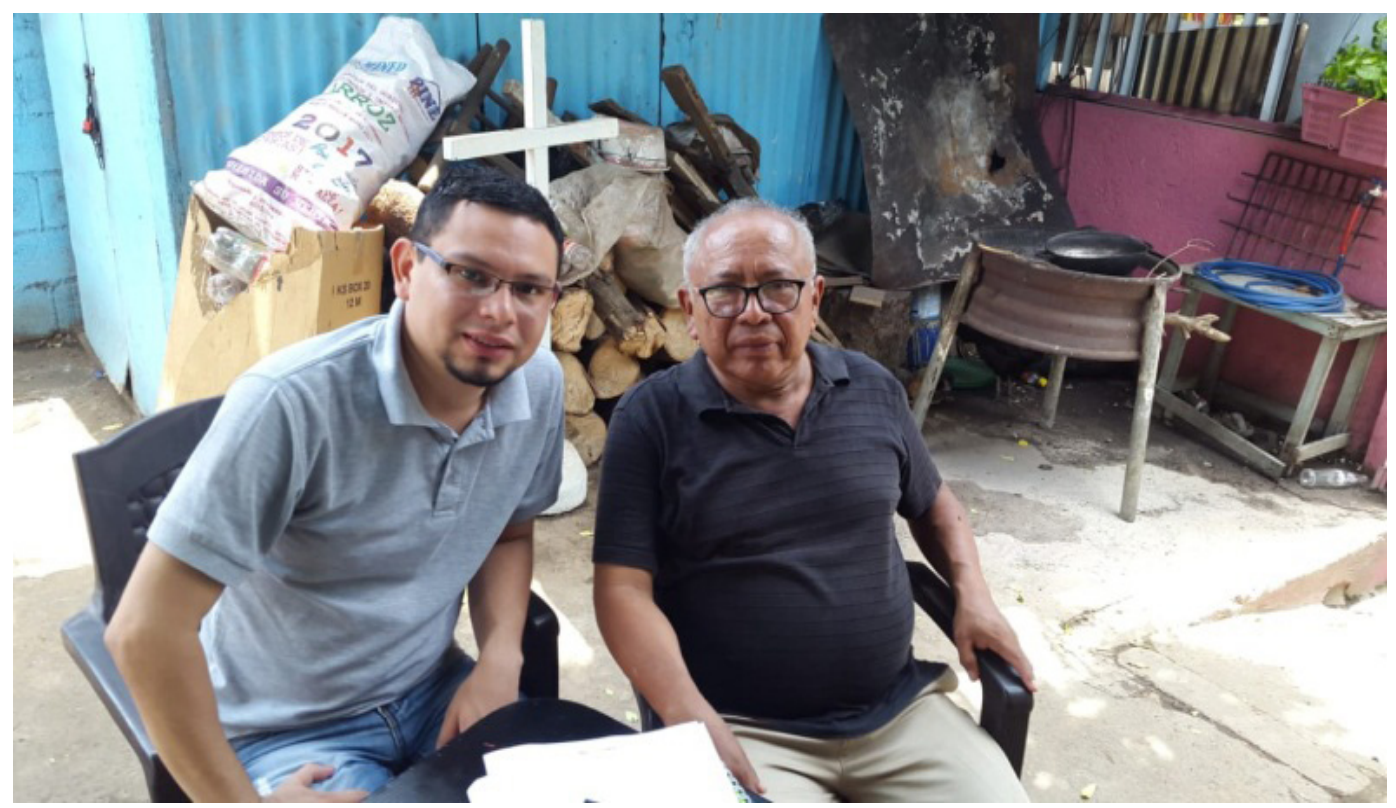

Figura 1. Entrevista realizada al profesor Francisco Roberto García.

Esto conllevó a la implementación de técnicas de investigación como la entrevista, en donde "el entrevistador busca información para su investigación y el entrevistado de alguna manera busca hacer pública su historia y sus puntos de vista" (Mariezkurrena, 2008, p.231); y también condujo a la construcción de historias de vida para profundizar más en el conocimiento del objeto de investigación. Las historias de vida consisten en "el relato de un narrador sobre su existencia a través del tiempo, intentando reconstituir los acontecimientos que vivió y transmitir la experiencia que adquirió" (Veras, 2010, p.144). 


\section{INNOVACIONES EDUCATIVAS}

Así también, para complementar la información recopilada mediante las fuentes orales, se realizó una revisión documental (Bernal, 2010; Katayama, 2014) mediante la cual se obtuvo información de fuentes escritas como documentos oficiales, libros de registros e informes que resguarda el colegio. Esta triangulación de fuentes de información tiene el propósito de determinar las intersecciones y coincidencias a partir de diferentes apreciaciones y fuentes informativas, eleva la confiabilidad de los datos y complementa lo que se ha recolectado a través de otras técnicas de investigación (Gurdián Fernández, 2007; Katayama, 2014).

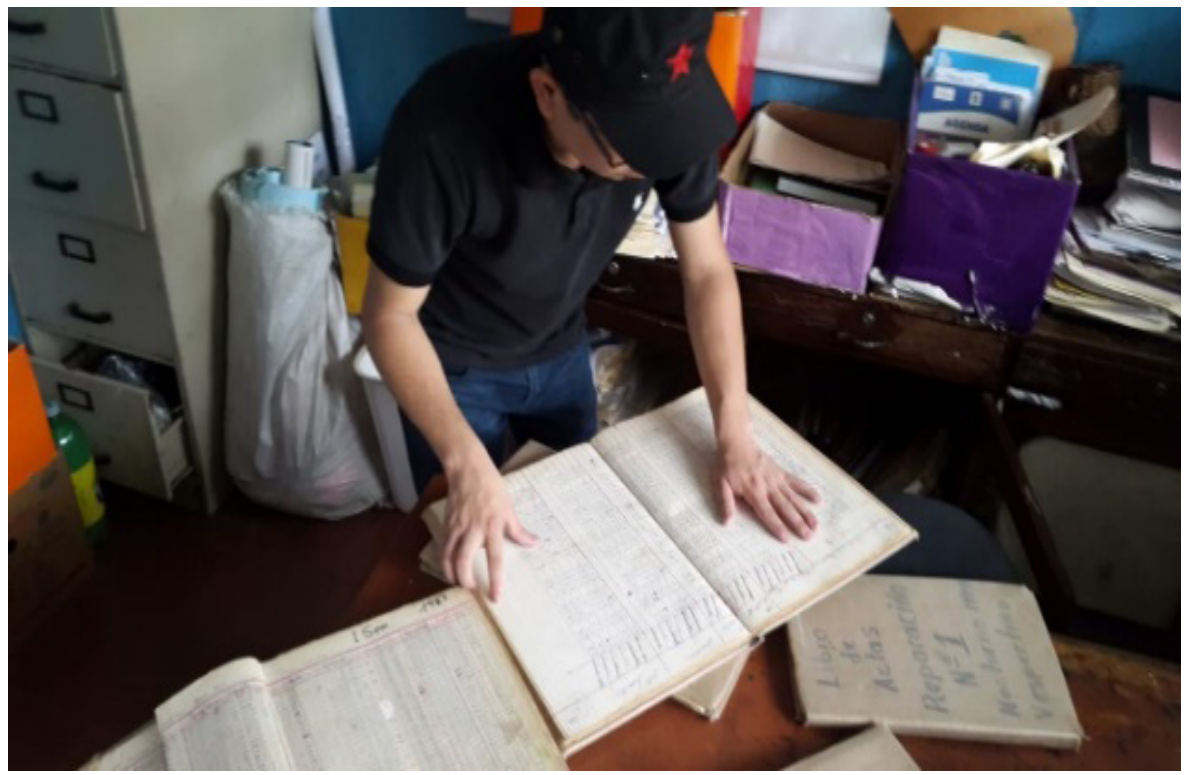

Figura 2. Revisión documental de libros de actas, registro e informes

En cuanto a la muestra seleccionada, ésta estuvo compuesta por personas que reunían "características acordes con el tema y variables que se necesitan estudiar" (Martínez González, 2007, p. 52), seleccionadas a través del muestreo no probabilístico por conveniencia en donde "el investigador selecciona a cada uno de los sujetos o unidades de la muestra de manera arbitraria" (Katayama, 2014, p.76). En total se realizaron once entrevistas, entre ellos a docentes activos, docentes jubilados, miembros de la comunidad, trabajadores administrativos y estudiantes egresados que tenían vastos conocimientos sobre la historia del colegio. También cabe agregar que la ubicación de los participantes se llevó a cabo mediante el método por bola de nieve (Katayama, 2014), en donde cada participante remitía al equipo de investigadores a otros sujetos que contenían información sobre el objeto de investigación.

La implementación de todos estos elementos metodológicos en el proceso investigativo, así como de instrumentos de investigación como diario de campo, fotografía 
y grabadora, permitieron recopilar una gran cantidad de información y reconstruir desde una perspectiva amplia la historia del Colegio Público de Esquipulas de Managua.

\section{Resultados}

\section{El primer colegio en la comunidad de Esquipulas.}

En la comunidad Esquipulas ubicada en el $\mathrm{km} \mathrm{111/2}$ carretera a Masaya, departamento de Managua, hacia el año 1976 aún no existía ningún centro educativo para niños, adolescentes y adultos. Las pocas personas que tenían las condiciones económicas para estudiar lo hacían viajando a comunidades aledañas en donde había escuelas que ofertaban la modalidad Primaria, principalmente al Colegio Josefa Toledo de Aguerri ubicado en la comunidad Gaspar García Laviana, a unos 2 km de distancia de la comunidad Esquipulas, el cual gozaba de alto prestigio a nivel de la comarca; y en el caso de los que tenían recursos para cursar sus estudios de Educación Secundaria, "iban a Managua a estudiar en el Colegio Cristóbal Colón o en el Colegio Ramírez Goyena, los cuales tenían un alto prestigio a nivel nacional" (F. García, 2018).

Así, conmovida por estas condiciones educativas precarias y por el alto índice de analfabetismo que había en la comunidad de Esquipulas, Esperanza Navas de Gadea, una profesora de origen chontaleña, Licenciada y Doctora en Química, docente de Educación Primaria en el Colegio Modesto Armijo de Managua, junto con su familia, emprenden un ambicionado proyecto socio educativo sin sospechar las dimensiones que este alcanzaría años después: la construcción de la primera escuela en la comunidad de Esquipulas.

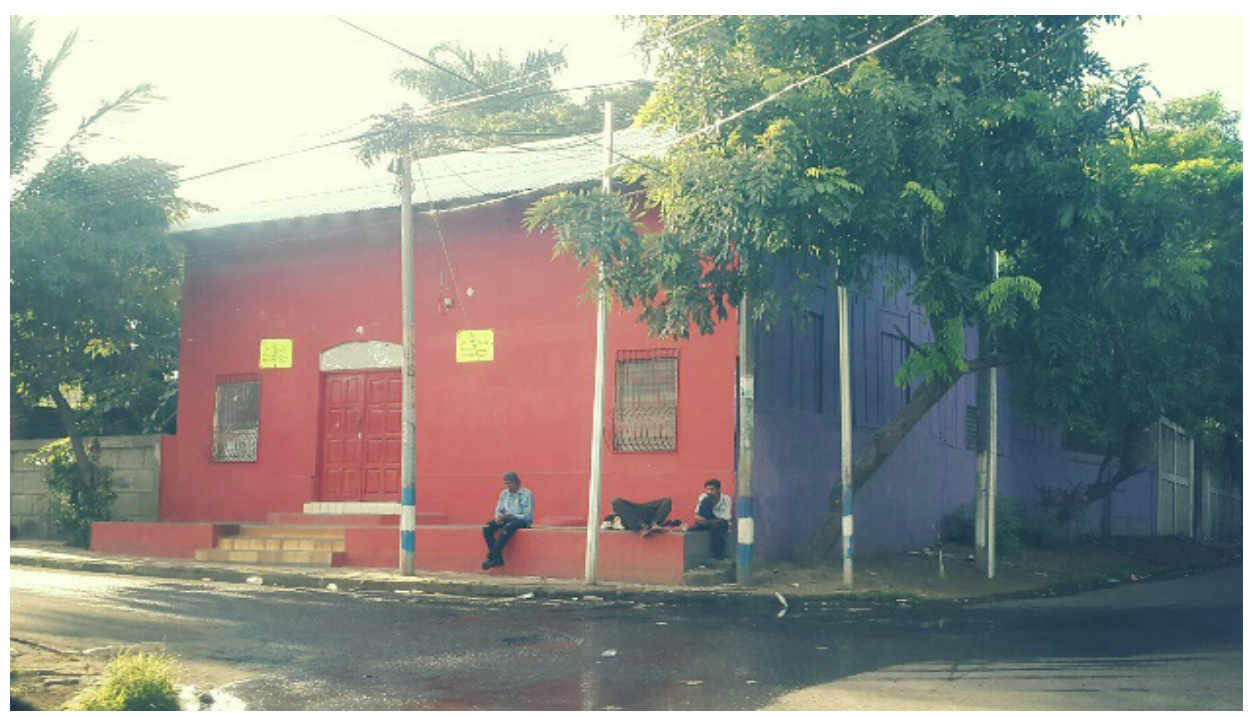

Figura 3. Casa comunal de Esquipulas en donde inició el CEDA 
Lo que hoy la comunidad de Esquipulas conoce como Instituto de Esquipulas aunque su nombre legítimo actualmente no es ese-, tuvo sus inicios hacia el año 1976 como Centro de Educación de Adultos (CEDA) - constituido jurídicamente como Ciclo Básico Nacional Nocturno de Esquipulas- cuando se apertura el primer y segundo ciclo en modalidad multigrado para niños en el turno vespertino, y el ciclo básico en el turno nocturno exclusivamente para personas trabajadoras de la comunidad, para entonces las instalaciones se ubicaron provisionalmente en la que fue la casa comunal de la comunidad durante muchos años. Nohemí Herrera (2018), quien fue estudiante en el CEDA, recuerda que en la casa comunal "era tuani recibir y dar clases porque había dos salas, en la grande estaban los adultos, y en la más pequeña los niños". En el CEDA la mayoría de los profesores eran familiares de la profesora Esperanza Navas de Gadea -la fundadora del colegio-, entre ellos se encontraban "el Ingeniero Humberto González, la Licenciada Mireya Ruiz, Diana Ruiz, Rosa María Gadea, el profesor Humberto González, la profesora Diana Ruiz y la profesora María Navas" (F. García, 2018), quienes impartían asignaturas como Matemática, Español, Estudios Sociales, Idiomas, Educación Física, Educación Musical, Educación Física y Conducta en el caso del Ciclo Básico, según lo reflejan los libros de registro académico del Colegio. Aurora Eugenia Mendoza Sandino, quien inició su experiencia como docente con la fundación del CEDA y quien laboró para tal institución educativa durante 35 años, recuerda que "en el CEDA la enseñanza era buena, había buenos maestros, calificados como normalistas, otros ya eran licenciados" (A. Mendoza, 2018).

Al ser éste el único centro de educación en la comunidad de Esquipulas y, en donde también estudiaban pobladores de comunidades aledañas, se experimenta una demanda creciente de matrícula estudiantil durante sus inicios la cual logra exceder la capacidad de las instalaciones de la casa comunal, surgiendo así la necesidad de ampliar sus instalaciones y consigo la oferta académica que hasta ese entonces tenía. Es así que por iniciativa de la profesora Esperanza Navas de Gadea, se comienza a gestionar con funcionarios del Ministerio de Educación - con quien tenía ella sostenía amistad-, con Cáritas de Nicaragua que era una institución de la Iglesia Católica de Nicaragua al servicio de lo social, y con miembros de la comunidad de Esquipulas para la construcción de este centro educativo en otro espacio geográfico en donde se solventaran las necesidades de ese momento. 


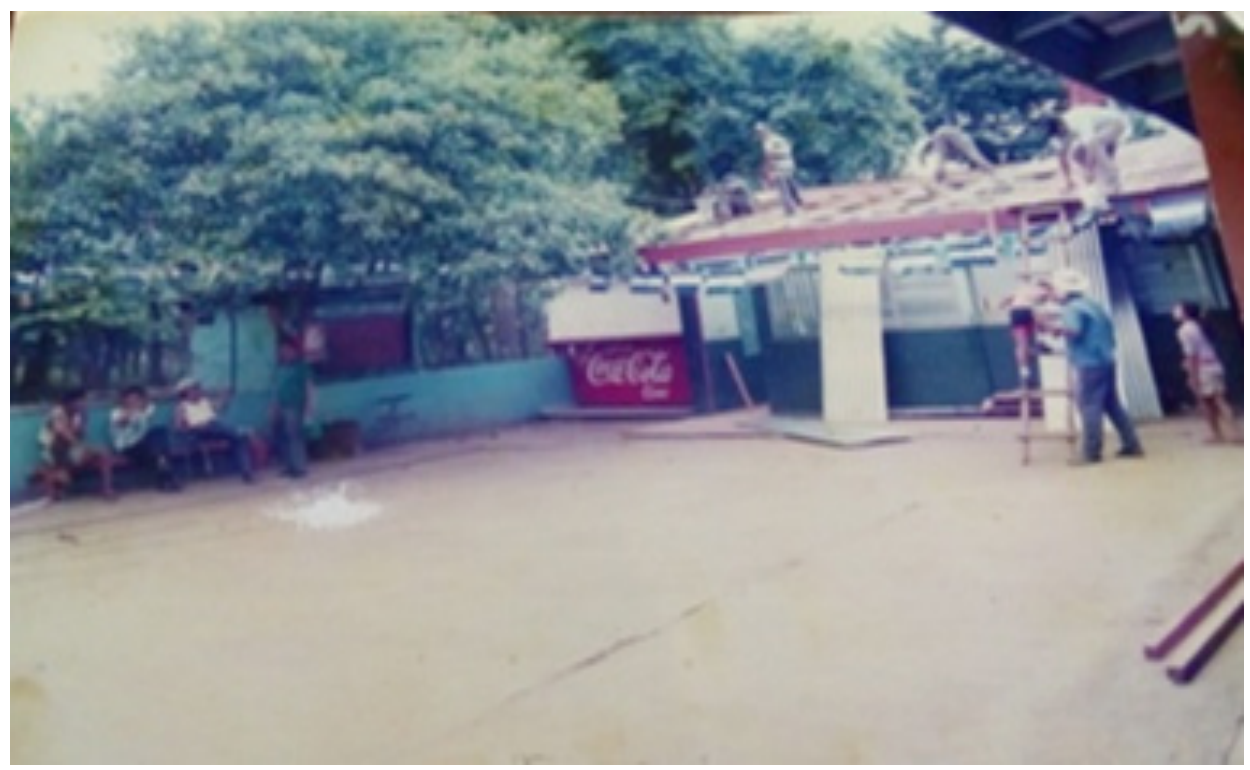

Figura 4. Pobladores colaboran en la construcción del colegio.

\section{La comunidad que construyó su propia escuela.}

Francisco Roberto García, conocido como el director Tito, cursó sus estudios de Educación Primaria y su Ciclo Básico de Secundaria en el CEDA, y fungió como director del mismo -años posteriores cuando este ya estaba constituido como Instituto- entre 1987-2008 y 2009-2013. Siendo en ese entonces estudiante, trabajador y poblador de la comunidad de Esquipulas, recuerda cómo se inició el traslado del CEDA y su construcción en la nueva propiedad:

Primeramente, ocurrió la donación del terreno por doña María Rivas, mamá de don David, el señor dueño del barcito del colegio. Luego vinieron las gestiones de doña Esperancita con el Ministerio de Educación y con Cáritas de Nicaragua. La construcción estaba a cargo de don Edgard Gómez y don Enrique García, miembros de la comunidad, pero toda la comunidad trabajó en la construcción del colegio voluntariamente, yo también trabajé, aún recuerdo a estudiantes del CEDA como batían mezcla (F. García, 2018).

La construcción del Ciclo Básico Nacional Nocturno de Esquipulas transcurría en el año 1978 cuando paralelamente en Nicaragua se gestaba una insurrección popular liderada por el Frente Sandinista de Liberación Nacional (FSLN) en contra de la dictadura somocista, lo cual agudizaba la carencia de recursos públicos para los servicios educativos, disponiendo así a las comunidades a buscar financiamientos alternos para la realización de proyectos sociales. Este fue un factor propicio para que, bajo la 
conducción de la profesora Esperanza Navas de Gadea, la comunidad de Esquipulas participara activamente en la construcción de su colegio.

Recuerdo que las mujeres de la comunidad llevaban comida y fresco como a las diez de la mañana a quienes trabajaban en la construcción. Caritas de Nicaragua apoyó a los albañiles con un medio pago. Y chavalos que estudiaban conmigo como Eddy Solórzano y Daniel Maltez iban junto a sus padres a plantarse al despacho del Vice Ministro de Educación, buscando apoyo para la construcción del colegio (F. García, 2018).

La profesora Esperancita buscó financiamiento para la construcción del colegio, le dieron los materiales y organizó a la comunidad para que cooperaran. Recuerdo cómo ella y miembros de la comunidad ayudaban cargando bloques, hierro, palas y otros materiales (K. Solórzano, 2018).

La construcción del Ciclo Básico Nacional Nocturno de Esquipulas en la nueva propiedad logró concretarse en 1978 con solamente un pabellón, tres secciones grandes y un amplio patio, atendiendo únicamente el primer y segundo ciclo de Educación Primaria en turno matutino y el tercer ciclo de Educación Secundaria en turno nocturno. Sin embargo, en 1979 con la apertura del cuarto ciclo se logra completar la Educación Secundaria y los estudiantes alcanzaban el grado de bachiller, y aumenta la matrícula estudiantil, por lo que la ampliación del colegio no quedaba allí. Con el triunfo de la revolución la ampliación de su infraestructura siguió siendo una necesidad y la participación de la comunidad siguió haciéndose notar. Nohemí Herrera recuerda que:

Cuando se estaba ampliando el colegio los mismos alumnos del nocturno apoyaron en la construcción del colegio, decían vamos a hacer un rojinegro, las mujeres apoyaban con la comida, y el ministerio daba algunos materiales para la construcción de las aulas (N. Herrera, 2018).

\section{Los nombres del Colegio, una larga travesía...}

Lo que en 1976 marcaba un hito en la comunidad de Esquipulas con el nombre de Ciclo Básico Nacional Nocturno de Esquipulas, hoy es una historia viviente con un enorme recorrido que, en Esquipulas y comunidades aledañas como Los Vanegas, San Antonio Sur, Gaspar García Laviana, Santo Domingo, Las Jagüitas, Ticuantepe, Las Cuarezma, La Hollada, entre otras, se conoce como Colegio Público de Esquipulas. Sin embargo, este tránsito tiene una larga travesía. 


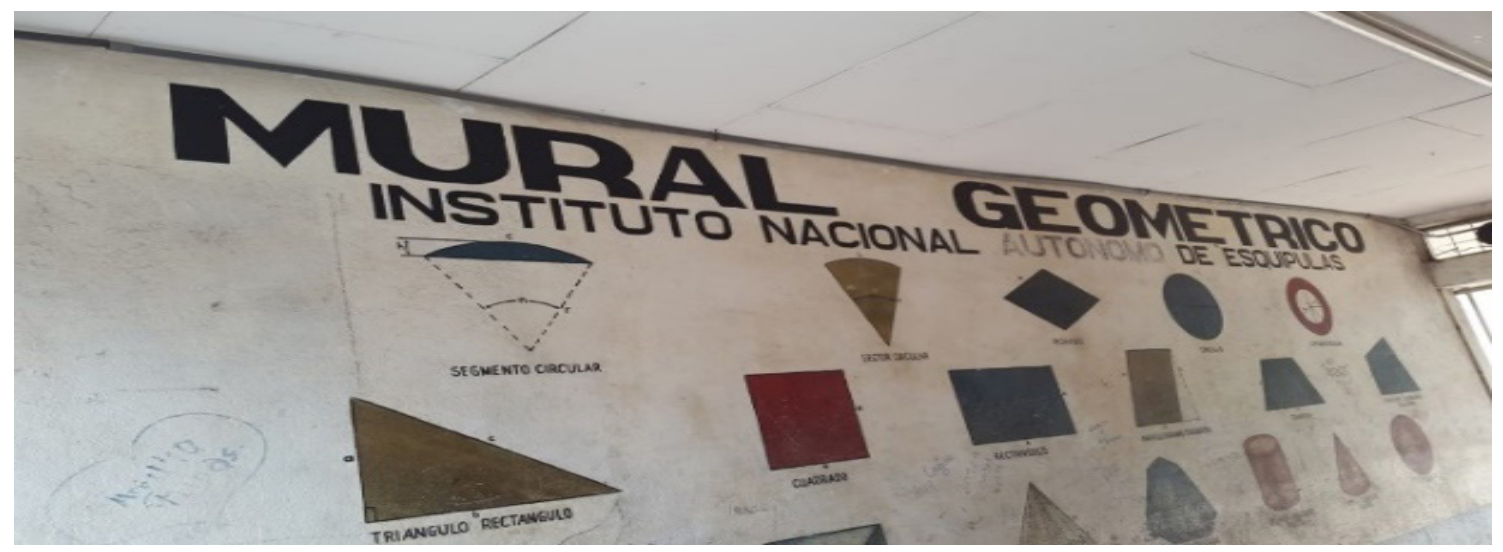

Figura 5. Mural del colegio correspondiente al año de 1997

La constitución de los nombres de los distintos Colegios Públicos de Nicaragua se ha caracterizado por sucesivos cambios que en su momento han tendido a generar confusión en la comunidad, estudiantado y profesorado. Esta larga travesía obedece a la repercusión de los acontecimientos y cambios sociopolíticos que ha experimentado el país, por ejemplo: con el fin de la dictadura somocista (1979), el triunfo de la Revolución Popular Sandinista (1979-1990), el periodo gobiernos neoliberales (19902006) y el retorno del gobierno sandinista en 2007. En el presente caso, lo que hoy se conoce con el nombre de Colegio Público de Esquipulas, comenzó a funcionar en 1976 con el nombre de Ciclo Básico Nacional Nocturno de Esquipulas, sin embargo, prontamente con el triunfo de la Revolución Popular Sandinista en 1979, el colegio tomaría otro nombre, porque, como explica el profesor Francisco Roberto García, "con el triunfo de la revolución los colegios y escuelas empezaron a ser nombrados oficialmente con nombres de héroes y mártires de la revolución. Entonces el CEDA pasó a llamarse Oscar Turcio Chavarría" (F. García, 2018). No obstante, de acuerdo con lo sostenido por F. García (2018), N. Herrera (2018) y D. Murillo (2018) y lo encontrado en las fuentes documentales examinadas (libros de actas, circulares, sellos, libros de registros, documentos oficiales y personales), se conoce que el colegio ocupó el nombre de Instituto Nacional Óscar Turcios Chavarría entre los periodos 1983-1994.

Con el advenimiento de los gobiernos neoliberales en 1990, los colegios también asumen nuevos nombres con el objetivo de borrar la memoria histórica de la revolución, pasando el colegio a adoptar primeramente el nombre de Instituto Autónomo Óscar Turcios Chavarría (1995-1996), luego con la autonomía escolar se llamó Instituto Nacional Autónomo de Esquipulas (1997-1998), Liceo Nacional Autónomo de Esquipulas (1999-2000) en el cual se pretendía incorporar clases técnicas, Instituto Público Autónomo Esquipulas (2001-2002), y en la última etapa tomó el nombre de Colegio Público Autónomo de Esquipulas (2003), mismo que ocuparía hasta el 2007 cuando retorna el gobierno sandinista y asume su nombre actual Colegio Público de Esquipulas. 


\section{El sueño del laboratorio de computación}

Uno de los sueños que persiguió con mucho ahínco el director Francisco Roberto García en sus veinticinco años de gestión administrativa, fue construir un laboratorio de computación, y el profesorado que compartió largo tiempo laboral con él también lo recuerdan: "nosotros queríamos un laboratorio, se luchó muchos años por ese proyecto, al final se hizo con mucho sacrificio, pero no duró" (N. Rizo, 2018), "ese era un sueño del director, y lo logró por un tiempo" (D. Murillo, 2018).

Efectivamente, la construcción de un laboratorio de computación era un proyecto ambicioso en medio del contexto precario en que se situaba la educación en los primeros años del presente siglo, sin embargo, ante la falta de apoyo de las autoridades del Ministerio de Educación Cultura y Deporte (MECD) de esos años, la comunidad seguía construyendo su colegio. Hacia el año 2005 se inaugura el laboratorio de computación con alrededor de 20 computadoras, en donde los estudiantes por primera vez tenían la oportunidad de utilizar este tipo de tecnología que en ese entonces era poco conocida. El profesor Francisco Roberto García lo recuerda de la siguiente manera:

Los alumnos recibían una hora teórica y luego una hora práctica. Los maestros de computación no eran nombrados por el Ministerio de Educación, así que los estudiantes aportaban una ayuda mínima de diez córdobas, incluso los maestros de computación lograron con ayuda de los alumnos integrar el internet en el laboratorio de computación (F. García, 2018).

Este proyecto de computación fue muy significativo para el estudiantado que procedía de comunidades rurales aledañas a Esquipulas en donde se carecía de este tipo de tecnología, y en el casco urbano eran pocos los hogares que tenía una computadora. Kathya García, estudiante del Colegio Público de Esquipulas entre 2006 y 2010, relata su experiencia al respecto:

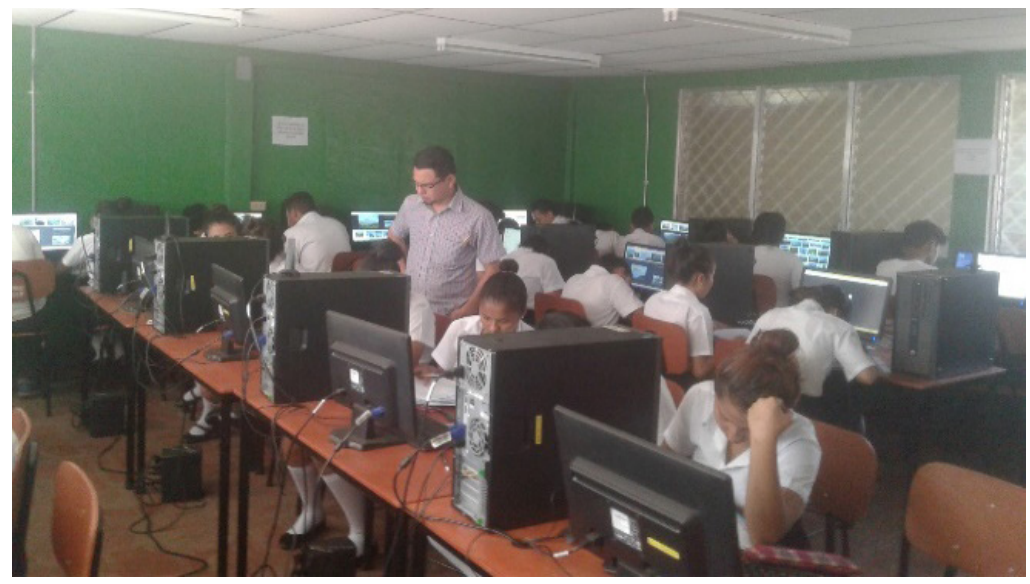

Figura 6. Aula TIC del Colegio Público de Esquipulas. 
En ese tiempo no era común que los jóvenes tuviéramos computadoras, y recibir clases de computación en el Instituto, aunque no fuera por mucho tiempo, fue muy significativo para mí. Yo adquirí conocimientos que todavía recuerdo y utilizo, ahí nos enseñaron mecanografía, a archivar documentos, quemar discos, hacer presentaciones en power point y hacer trabajos en Word, y esto me sirvió mucho, porque años después yo entré a estudiar al CECNA3 y ya conocía esas cosas (K. García, 2018).

Y aunque el proyecto fue efímero -se mantuvo hasta mediados del año 2006- a causa del fin de la autonomía escolar y por la falta de recursos económico, este nunca dejó de existir en el imaginario de la comunidad educativa. Oportunamente, en el año 2016 por gestiones de la profesora Lidia Escobar Escalante, directora del Colegio Esquipulas desde 2014 hasta la actualidad (2018), se inició la construcción de un sofisticado laboratorio de computación con alrededor de 25 computadoras con acceso a internet, aire acondicionado, data show y un profesor de la especialidad, para ofertar su servicio a la comunidad educativa del Colegio:

Efectivamente, hicimos gestiones para que el Instituto tuviera su aula TIC, la construcción demoró un buen tiempo, pero a inicios del año 2016 era una realidad. Ahora los estudiantes hacen sus investigaciones y los profesores imparten sus clases apoyándose de la tecnología (Escobar, 2018).

\section{Las personalidades del Colegio Público Esquipulas en la nostalgia de sus estudiantes}

En las memorias del Colegio Público Esquipulas se encuentran distintas personalidades académicas y administrativas recordadas con mucha nostalgia entre el profesorado, estudiantado y comunidad, y sus directores Esperanza Navas de Gadea (1976-1980), Lea Escoto (interina durante tres meses en 1980), Miriam Viales (1980-1983), Julio César Cruz Chávez (1984-1987), Francisco Roberto García (1987-2008); Leonel Ríos (2008-2009), Francisco Roberto García (2009-2013), y Lidia Escobar (2014-actualidad), estas son las personas que han llevado a cabo este sueño y una responsabilidad social con dimensiones históricas. Así también, entre la centena de profesores que ha tenido el Colegio Público de Esquipulas, las generaciones egresadas aún recuerdan con nostalgia a profesores como:

El profesor Narciso Rizo: Fue docente del Colegio Público de Esquipulas por veintiocho años -actualmente jubilado-, e impartió asignaturas como Lengua y Literatura, Convivencia y Civismo, y Orientación, Técnica y Vocacional, entre otras. Comúnmente es conocido por las distintas generaciones con el seudónimo "chicho", y se caracterizó por su carisma, accesibilidad, flexibilidad y amistad, así también, por su amabilidad con el estudiantado: "Él es experto en cambiar los nombres y apellidos de 


\section{INNOVACIONES EDUCATIVAS}

los estudiantes. Era un profesor muy divertido, pero cuando vos le preguntabas algo sobre el contenido él respondía muy acertadamente, lo dominaba" (O. García, 2018).

El profesor Mauricio Valdizón: Ha impartido por muchos años las asignaturas Ciencias Naturales, Química y Biología, es reconocido por sus convicciones, principios y valores patrióticos y sociales, y por estar comprometido con el cuido del medio ambiente y con la formación de ciudadanos conscientes de su realidad social.

"Él es un buen profesor, me dejó muchas lecciones no solo de Ciencias Naturales, Química y Biología. Recuerdo que él nos enseñó que el Himno Nacional no se debía aplaudir y que limpiarse los oídos con hisopo no es correcto" (O. García, 2018).

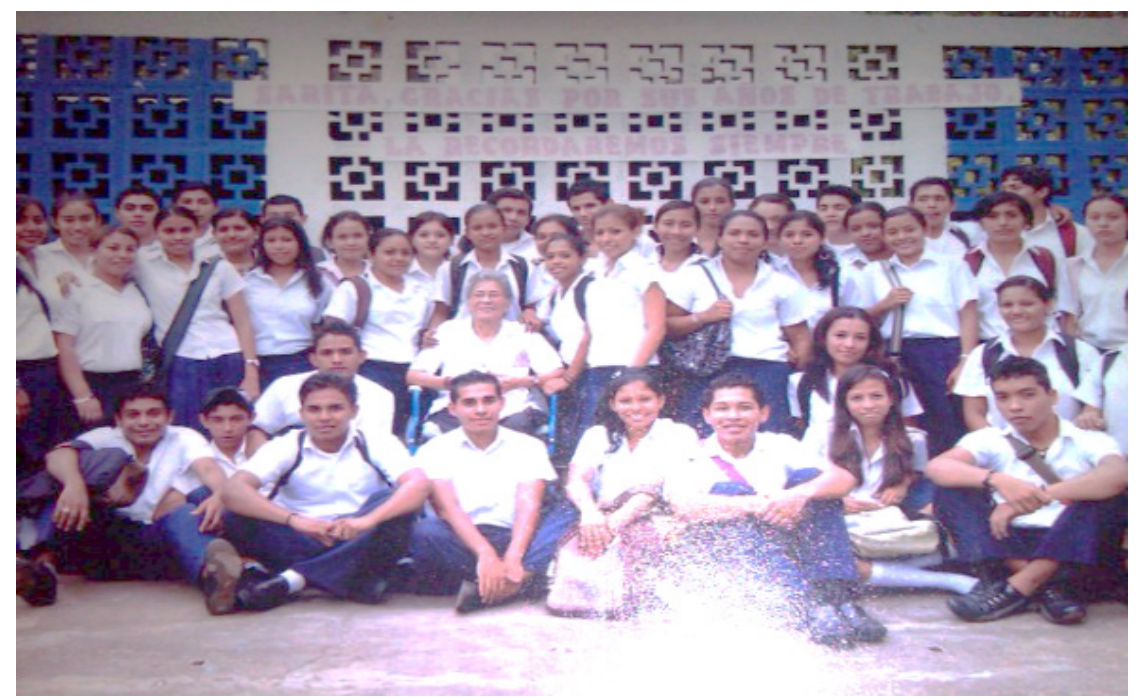

Figura 7. La generación 2010 dedicó su promoción a la secretaria académica Sarita Pérez

Las personalidades administrativas también figuran en la memoria histórica del colegio, tal es el caso de Sarita Pérez quien fungió como secretaria académica por más de 25 años, y es recordada como una persona trabajadora y servicial, que pese a sus limitantes físicas nunca evadió sus responsabilidades laborales. Sara López Castillo, trabajó como Bibliotecaria del colegio entre 2001 y 2017, recuerda a doña Sarita Pérez de la siguiente manera: Era una persona servicial, con mucha disposición con aquellas personas que solicitaban certificados de notas, boletines, gestiones de los diplomas de bachilleres. Era muy ordenada, responsable y sonriente. Resolvía a lo inmediato a quienes solicitaban algún servicio con urgencia (S. López, 2018)

Pedro Estrada: Es conocido cariñosamente como "don Pedrito o don Peter". Laboró en el colegio por más de 30 años como guarda de seguridad. Se le veía siempre en el portón del colegio dándoles entrada a los estudiantes un cuarto de hora antes 
de iniciar las clases, y con voz fuerte y autoritaria no vacilaba en retener a aquellos estudiantes que no portaban el uniforme escolar correctamente:

"Era tranquilo y exigente, y su decisión se respetaba. No dejaba entrar a alumnas con faldas cortas, calcetas abajo, ni muy pintadas, ni a varones con cabello largo, camisa de fuera y sin escarapelas. Era una forma muy buena de controlar la disciplina de los estudiantes" (K. García, 2018).

"En 1993 nuestra generación reconoció su labor al dedicarle nuestra promoción a él. Siempre lo recordamos por su compromiso con la disciplina y resguardo de los estudiantes" (I. Sánchez, 2018).

\section{Discusión y conclusiones}

La memoria histórica del Colegio Público Esquipulas demuestra que la historia de los centros educativos está muy ligada a las transformaciones políticas que ha experimentado el país en las distintas décadas, sin los cuales, no se puede obtener una comprensión objetiva de todo el proceso histórico del mismo. Los resultados del estudio también demuestran que los pocos recursos públicos que se le asignaron al colegio no fue una limitante para su funcionamiento, puesto que, desde su fundación, su comunidad ha participado activamente en su construcción.

Así también, cabe destacar la importancia del uso de técnicas orales para la recopilación de información, éstas permitieron conocer desde distintas perspectivas todo lo concerniente a la historia del colegio, y la recopilación de fuentes escritas permitieron corroborar y situar en el tiempo con más precisión, los hallazgos obtenidos mediante las fuentes orales. Esta triangulación de información fue propicia para brindar aportes objetivos en cuanto al proceso histórico del colegio.

Finalmente, es de hacer notar que la recuperación de la memoria histórica de los centros educativos es una tarea esencial que no solamente ha de ser ejercida por investigadores provenientes de disciplinas como la historia y la antropología, sino que esta tarea también incumbe al profesorado de ciencias sociales, quienes pueden utilizar estos aportes con fines didácticos para el fortalecimiento de la identidad local e institucional, y para la promoción de valores como el cuido del patrimonio histórico de los centros educativos. 


\section{Lista de referencias}

Abraham, M. (2015). El proceso de investigación. En Introducción a los métodos y técnicas de investigación. Selección de lecturas (pp. 118-127). Cuba: Editorial Universitaria Félix Varela.

Bernal, C. (2010). Metodología de la investigación. (3ra ed.). Colombia: Pearson Educacación.

Escobar, Lidia. (2018). Entrevista de A, Díaz Pérez [Cinta de audio]. Proyecto Historia del Colegio Público Esquipulas, Managua.

Fernández, S. y Rivera, Z. (2015). El paradigma cualitativo y su presencia en las investigaciones de la BCI. En Introducción a los métodos y técnicas de investigación. Selección de lecturas (pp. 88-117). Cuba: Editorial Universitaria Félix Varela.

García, Francisco. (2018). Entrevista de A, Díaz Pérez [Cinta de audio]. Proyecto Historia del Colegio Público Esquipulas, Managua.

García, Kathya. (2018). Entrevista de A, Díaz Pérez [Cinta de audio]. Proyecto Historia del Colegio Público Esquipulas, Managua.

García, Oscar. (2018). Entrevista de A, Díaz Pérez [Cinta de audio]. Proyecto Historia del Colegio Público Esquipulas, Managua.

Gurdián Fernández, A. (2007). El Paradigma Cualitativo en la Investigación SocioEducativa. San José: IDER.

Hernández, R., Fernández, C. y Baptista, M. (2014). Metodología de la Investigación. (6ta ed.). México: McGRAW-HILL.

Herrera, Nohemí. (2018). Entrevista de A, Díaz Pérez [Cinta de audio]. Proyecto Historia del Colegio Público Esquipulas, Managua.

Katayama, R. (2014). Introducción a la Investigación Cualitativa: Fundamentos, métodos, estrategias y técnicas. Perú: Universidad Inca Garcilaso de la Vega.

López, Sara. (2018). Entrevista de A, Díaz Pérez [Cinta de audio]. Proyecto Historia del Colegio Público Esquipulas, Managua. 
Mariezkurrena, D. (2008). La historia oral como método de investigación histórica. Revista Guillermo de Uztariz, (23/24):227-233.

Martínez González, R. (2007). La Investigación en la Práctica Educativa: Guía Metodológica de Investigación para el Diagnóstico y Evaluación en los Centros Docentes. Madrid: Ministerio de Educación y Ciencia. Dirección General de Educación, Formación Profesional e Innovación Educativa.

Mendoza, Aurora. (2018). Entrevista de I, Silva [Cinta de audio]. Proyecto Historia del Colegio Público Esquipulas, Managua.

Murillo, Delfa. (2018). Entrevista de A, Díaz Pérez [Cinta de audio]. Proyecto Historia del Colegio Público Esquipulas, Managua.

Rizo, Narciso. (2018). Entrevista de A, Díaz Pérez [Cinta de audio]. Proyecto Historia del Colegio Público Esquipulas, Managua.

Rodríguez, A., Luque, R. y Navas, A. (2014). Usos y beneficios de la Historia Oral. Reidocrea, (3):193-200.

Sánchez, Ivania. (2018c). Entrevista de A, Díaz Pérez [Cinta de audio]. Proyecto Historia del Colegio Público Esquipulas, Managua.

Solórzano, Karla. (2018). Entrevista de I, Silva [Cinta de audio]. Proyecto Historia del Colegio Público Esquipulas, Managua.

Veras, E. (2010). Historia de vida ¿Un método para las ciencias sociales? Cinta moebio, (39):142-152. 